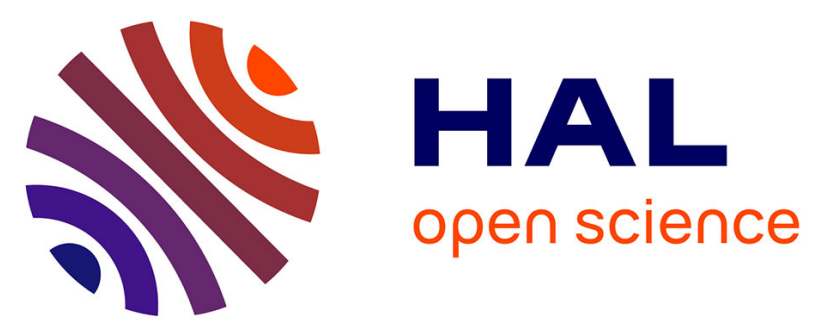

\title{
Personalization of a Cardiac Electromechanical Model using Reduced Order Unscented Kalman Filtering from Regional Volumes
}

Stéphanie Marchesseau, Hervé Delingette, Maxime Sermesant, Rocio Cabrera Lozoya, Catalina Tobon-Gomez, Philippe Moireau, Rosa Maria Figueras I

Ventura, Karim Lekadir, Alfredo Hernandez, Mireille Garreau, et al.

\section{To cite this version:}

Stéphanie Marchesseau, Hervé Delingette, Maxime Sermesant, Rocio Cabrera Lozoya, Catalina TobonGomez, et al.. Personalization of a Cardiac Electromechanical Model using Reduced Order Unscented Kalman Filtering from Regional Volumes. Medical Image Analysis, 2013, 17 (7), pp.816-829. 10.1016/j.media.2013.04.012 . hal-00819806

\section{HAL Id: hal-00819806 https://hal.inria.fr/hal-00819806}

Submitted on 2 May 2013

HAL is a multi-disciplinary open access archive for the deposit and dissemination of scientific research documents, whether they are published or not. The documents may come from teaching and research institutions in France or abroad, or from public or private research centers.
L'archive ouverte pluridisciplinaire HAL, est destinée au dépôt et à la diffusion de documents scientifiques de niveau recherche, publiés ou non, émanant des établissements d'enseignement et de recherche français ou étrangers, des laboratoires publics ou privés. 


\title{
Personalization of a Cardiac Electromechanical Model using Reduced Order Unscented Kalman Filtering from Regional Volumes
}

\author{
S. Marchesseau - H. Delingette - M. Sermesant - R. Cabrera-Lozoya * \\ C. Tobon-Gomez · P. Moireau · R.M. Figueras i Ventura · K. Lekadir \\ A. Hernandez · M. Garreau - E. Donal · C. Leclercq · S.G. Duckett · \\ K. Rhode - C.A. Rinaldi - A.F. Frangi - R. Razavi - D. Chapelle · \\ N. Ayache
}

Received: date / Accepted: date

\begin{abstract}
Patient-specific cardiac modelling can help in understanding pathophysiology and therapy planning. However it requires to combine functional and anatomical data in order to build accurate models and to personalize the model geometry, kinematics, electrophysiology and mechanics. Personalizing the electromechanical coupling from medical images is a challenging task. We use the Bestel-Clément-Sorine (BCS) electromechanical model of the heart, which provides reasonable accuracy with a reasonable number of parameters (14 for each ventricle) compared to the available clinical data at the organ level. We propose a personalization strategy from cine MRI data in two steps. We first estimate global parameters with an automatic calibration algorithm based on the Unscented Transform which allows to initialize the parameters while matching the volume and pressure curves. In a second step we locally personalize the contractilities of all AHA (American Heart Association) zones of the left ventricle using the Reduced Order Unscented Kalman
\end{abstract}

\section{S. Marchesseau}

INRIA, Asclepios Research Project, Sophia Antipolis, France Tel.: +334-92-387151

E-mail: Stephanie.Marchesseau@inria.fr

N. Ayache $\cdot$ R. Cabrera-Lozoya $\cdot$ H. Delingette $\cdot$ M. Sermesant INRIA, Asclepios Research Project, Sophia Antipolis, France

C. Tobon-Gomez · R.M Figueras i Ventura · K. Lekadir · A.F. Frangi

CISTIB, Universitat Pompeu Fabra, Barcelona, Spain

K. Rhode · C.A. Rinaldi · S.G.Duckett · R.Razavi

King's College London, Division of Imaging Sciences \& Biomedical Engineering, St. Thomas' Hospital, London, UK

C. Leclercq · E. Donal · A. Hernandez · M. Garreau INSERM, Université de Rennes, FRANCE

P. Moireau · D. Chapelle

INRIA, MACS Project-Team, Saclay Ile-de-France, France
Filtering on Regional Volumes. This personalization strategy was validated synthetically and tested successfully on eight healthy and three pathological cases. Computer Model - Cardiac Mechanics - Medical Images - Patient-Specific Models - Regional Volumes Reduced Order Unscented Kalman Filtering

\section{Introduction}

Understanding pathologies, planning a therapy, training for a surgery or selecting suitable patients for a treatment are challenges that cardiologists face everyday. For instance, Cardiac Resynchronization Therapy (CRT), which releases heart failure with a pacemaker, is still difficult to predict in spite of many advances (Chung et al, 2008; Cazeau et al, 2001), and fails in $30 \%$ of the treated cases. To ease their tasks, a significant amount of image data is now available. One goal of computational cardiology is to use cardiac imaging data to build patient-specific electromechanical models of the heart that can then be used to better analyze and simulate cardiac function (Smith et al, 2011; Sermesant et al, 2012).

In order to reach such a goal, several components are mandatory. Firstly, an accurate anatomical model has to be extracted from the image data to represent the heart geometry. Recently, various approaches have been proposed to automatically segment cardiac images from different modalities, such as echocardiography (Butakoff et al, 2011; Zhu et al, 2010), computed tomography (CT) (Ecabert et al, 2011; Zheng et al, 2008) and magnetic resonance imaging (MRI) (Petitjean and Dacher, 2011; Schaerer et al, 2010). In particular, MRI has the advantage of providing a good image quality noninvasively and without radiation. In this paper, we extract personalized geomet- 
rical models from MRI by using a 3D Active Shape Model approach by Tobon-Gomez et al (2012). The estimated motion of the myocardium is also needed to build patient-specific simulations. This motion can be estimated from sequences of MRI data using various algorithms (McLeod et al, 2012; Mansi et al, 2011; De Craene et al, 2012a). Then, an electrophysiological model has to be considered in order to simulate the electrical wave propagation within the myocardium which leads afterwards to the contraction of the muscle cells. Since Hodgkin and Huxley (1952), several approaches have been developed to describe the electrophysiology, from biophysical models that simulate ionic interaction (Noble, 1962), to phenomenological models based on reaction-diffusion equations (FitzHugh, 1961; Aliev and Panfilov, 1996) and recent adaptations of those (Clayton et al, 2011; Pashaei et al, 2011; Relan et al, 2011). They differ in the scale they use and their complexity in terms of computation times and number of parameters. These models have to be personalized from endocardial mappings and electrocardiograms (Relan et al, 2011) before serving as input to the mechanical simulation.

Finally, the electromechanical coupling itself has a high impact on the resulting simulation. For the past 20 years, the creation of such models has been the focus of many research teams and various models have been proposed (Humphrey et al, 1990; Hunter et al, 1997; Nash, 1998; Bestel et al, 2001; Sachse, 2004). In this paper, the Bestel-Clément-Sorine cardiac model (Bestel et al, 2001), further improved by (Chapelle et al, 2012b) is used. It shows a good compromise between complexity and accuracy (Marchesseau et al, 2012b), and a good predictive power for CRT under different pacing conditions (Sermesant et al, 2012). The personalization of such mechanical models has been tackled by different approaches. Variational assimilation methods (Delingette et al, 2012; SainteMarie et al, 2006) were used to locally estimate the active parameters (contractility, contraction and relaxation rates), Sequential Quadratic Programming (Wang et al, 2009) to estimate the passive material stiffness, while Reduced Order Unscented Kalman Filtering was aimed at estimating the contractility parameters (Chabiniok et al, 2011; Moireau and Chapelle, 2011).

These methods have already led to promising results to estimate some model parameters from heart motion and therefore open the possibility to help cardiologists in planning their therapy. In this paper we chose to tackle this issue in a different way. We propose to estimate the regional contractilities of the left ventricle based on measured Regional Volumes. Using regional volumes has the advantage of filtering registration errors by only considering displacements orthogonal to the surface thus accounting for the aperture problem. Moreover, automatic estimation of regional volumes is already available in commercial software which may help to streamline the personalization process. We apply this strategy on a database consisting of eight healthy subjects and three heart failure patients. To this end, we first calibrate the mechanical model using the Unscented Transform method proposed in (Marchesseau et al, 2012a) from the left volume curve that we extract from the motion registered from cine MRI data. Then we apply the Reduced Order Unscented Kalman Filtering (Moireau and Chapelle, 2011) - using the Verdandi library ${ }^{1}$ - on the regional volumes measured on the LV endocardium. This strategy was successfully tested synthetically for contractility estimation and scar tissue detection, and on real cases. Therefore, the use of regional volumes to personalize the regional contractilities proved to be efficient and robust and testing the full pipeline on 8 healthy and 3 pathological cases enabled to draw preliminary conclusions on parameter specificity. In this respect and to the best of our knowledge, this study exhibits the largest cohort of healthy and pathological cases for personalized cardiac models to date.

\section{Materials and Methods}

\subsection{Data Acquisition}

The proposed study was performed on 3 pathological and 8 healthy cases. Data were acquired either at INSERM (Institut National de la Santé et de la Recherche Médicale, Université de Rennes 1, France), or at the Division of Imaging Sciences \& Biomedical Engineering at King's College London, UK, as part of studies that were ethically approved. Written informed consent was obtained from all human subjects and the studies were performed with all appropriate institutional human subjects approvals. All datasets consist of sequences of cine SSFP MRI with a spatial resolution of $1.5 \times 1.5 \times 7 \mathrm{~mm}^{3}$ for the healthy subjects and $1.25 \times 1.25 \times 10.8 \mathrm{~mm}^{3}$ for the pathological cases. Moreover, an intra-operative electrophysiological study (EnSite) was performed on all pathological cases.

\subsubsection{Real cases study}

All heart failure patients were selected for Cardiac Resynchronization Therapy based on criteria from the

1 Verdandi is an opensource data assimilation library available at http://verdandi.gforge.inria.fr/. (Chapelle et al, 2012a) 
New York Heart Association (NYHA) class III-IV. Patients had a small ejection fraction $(\mathrm{EF})<35 \%$ and a prolonged QRS on electrocardiogram $>120 \mathrm{~ms}$.

This study also includes extensive multi-modality imaging of volunteers from which 8 healthy cases were used. Volunteers were aged $28 \pm 5$ years, without clinical history of cardiac diseases. This database was made available to the research community for 1st Cardiac Motion Analysis (cMAC) challenge held at STACOM'2011, see (Tobon-Gomez et al, In Press) for details regarding the data acquisition of this study.

\subsubsection{Clinical Data Pre-Processing}

Three different steps are needed before any mechanical personalization can be performed: extraction of the myocardium geometry, estimation of the patient's cardiac motion and personalization of the electrophysiological propagation.

\section{Geometry Personalization}

We first extract a biventricular anatomy from SSFP MRI datasets using the segmentation algorithm implemented in (Tobon-Gomez et al, 2012). This semiautomatic segmentation technique is based on a deformable model and allows manual deformations if final corrections are needed. We generate a volumetric binary image from this biventricular surface (see Fig. 1) after interpolation and resampling on the z-axis for a higher resolution mesh. The Marching Cubes algorithm (Lorensen and Cline, 1987) is then used to extract a polygonal surface. ReMesh (Attene and Falcidieno, 2006) enables to smooth and ensure a manifold triangular mesh while Tetgen ${ }^{2}$ and Netgen ${ }^{3}$ generate the tetrahedral meshes (see Fig. 1) with approximately 14000 nodes. The sensitivity of the mesh resolution on the resulting motion was studied and enabled to conclude that this resolution is a good trade-off between computation times and accuracy with respect to the available data.

In addition, fibre directions were created synthetically as proposed by Streeter (Streeter, 1979). It is derived from both histological and DTI studies (Streeter, 1979; Hsu and Henriquez, 2001) and it has been extensively validated for cardiac simulation (Bayer et al, 2012). The elevation angle was varied (with respect to the short axis plane) across the myocardium wall from $-70^{\circ}$ on the epicardium to $0^{\circ}$ at mid-wall to $+70^{\circ}$ on the endocardium. This choice influences the global motion of the myocardium and was therefore set after a trial and error approach. In

\footnotetext{
${ }^{2}$ http://tetgen.berlios.de

${ }^{3} \mathrm{http}: / /$ www.hpfem.jku.at/netgen
}

the myocardium, the angle was interpolated using the distance from the selected node to the closest surface. Alternatively, a way of creating the fibres could be to map a statistical atlas of human cardiac fibres such as (Lombaert et al, 2011) onto the patient geometry. This would allow to include real patient data better than with predefined synthetic angles. However, statistical fibre models have not yet been validated for cardiac simulation.

\section{Motion Estimation}

Motion estimation was performed from cine MRI data using an automatic registration algorithm: the Time Diffeomorphic Free Form Deformation (TDFFD) algorithm, which has been tested on 3DUS datasets (De Craene et al, 2012a) and 3DTAG MRI datasets (De Craene et al, 2012b). The TDFFD algorithm optimizes a 4D velocity field parametrized by B-Spline spatiotemporal kernels.

With the transformation obtained from the registration, the static volumetric mesh was deformed and local or regional indices were computed. Due to its temporal consistency, this technique computes smooth volumetric meshes which is highly desirable for our application.

Resulting meshes were compared to the images in Fig.2 at end-diastolic phase (frame used for the segmentation) and end-systolic phase. We noticed
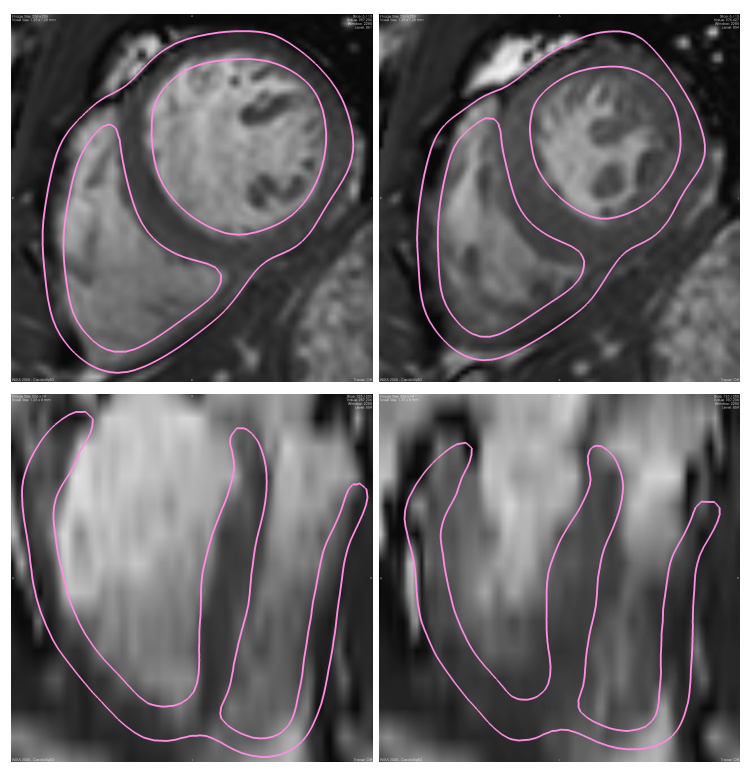

Fig. 2: Estimation of the motion of Volunteer \#1 (from left to right): end-diastolic frame, end-systolic frame.

from Fig.1 and Fig.2 that information on the basal area is very incomplete. Since we cannot estimate thoroughly the real movement in this area, we ignored the 

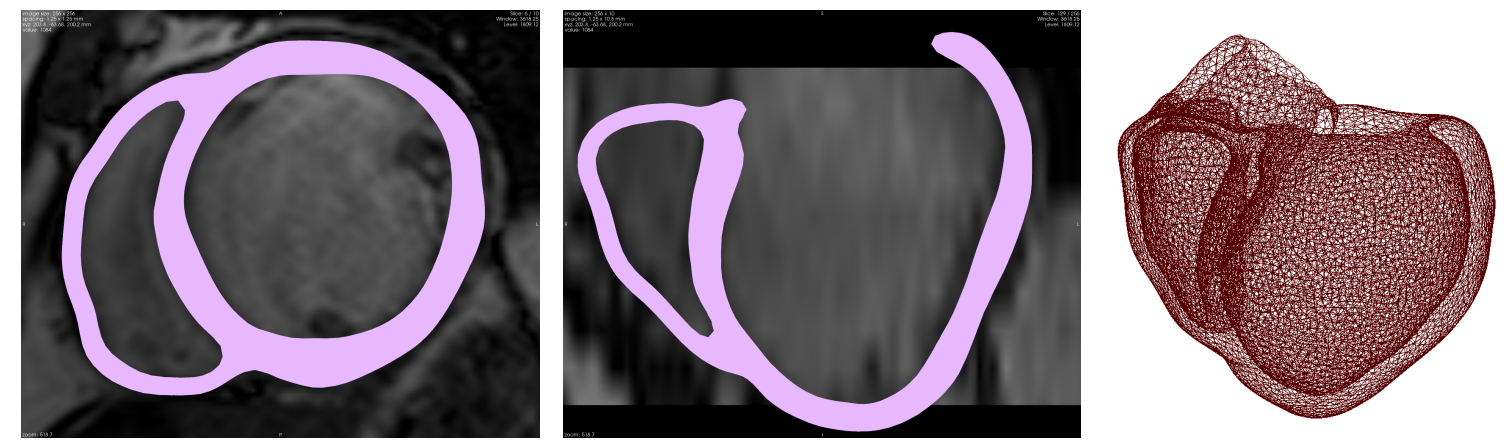

Fig. 1: Modeling the anatomy of patient Case \#1 (from left to right): extracting geometry from MR datasets, generating volumetric meshes.

observations extracted from the registration related to this area when personalizing the model. This results in smaller regional volume zones (see Section 2.2.3) but this does not alter the convergence of the algorithm. Moreover this prevents unrealistic parameters to be estimated without influencing the motion drastically.

\section{Electrophysiology Personalization}

A large number of electrophysiological models for cardiac cell action potential have been developed, ranging from models at the cellular to those at the sub-cellular scale. They can be divided into three categories:

- Biophysical models. Are the most complete because they model ionic currents. Nonetheless, their high complexity, computational cost and lack of observability of their parameters make them less suitable for parameter estimation from clinical data.

- Phenomenological models. Are based on PDEs and present an intermediate level of complexity and computational cost.

- Generic models. Are the least complex set of models and represent simplified action potentials or depolarization times. Eikonal models belong to this category and model the propagation of the time at which a given point is depolarized.

To simulate the electrophysiological pattern of activity, an Eikonal equation was used. The model was then solved for the depolarization time $T_{d}$ at each point of the mesh:

$v \sqrt{\nabla T_{d}^{t} \mathbf{D} \nabla T_{d}}=1$.

The local electrical conduction velocity is $v$ and $\mathbf{D}$ is the anisotropic conductivity tensor defined as

$\mathbf{D}=(1-r) \mathbf{f} \otimes \mathbf{f}+r \mathbf{I}$

where $\mathbf{f}$ is the fibre orientation, $r$ the anisotropy ratio and $\mathbf{I}$ the identity matrix.
The solution of this electrophysiological model was found using a Multi-Front Fast Marching Method (Sermesant et al, 2007). For healthy cases no subject specific electrophysiological data were acquired and therefore standard values were assumed (conduction velocity of $90 \mathrm{~cm} / \mathrm{s}$ and anisotropic ratio of 0.1 ). The personalization of this model requires to specify the onset of the electrical propagation (manually defined as areas corresponding to the extremities of the Purkinje network, on both endocardiums close to the apex).

For pathological cases, intracardiac non contact mappings allowed to estimate the time and localization of the onset of depolarization as well as electrical conductivities (see (Relan et al, 2011)). For the personalization, the Mitchell-Schaeffer (Mitchell and Schaeffer, 2003) biophysical model is used. It simulates the evolution of the transmembrane potential as a function of simplified inward and outward currents. An example of personalized electrophysiology is given in Fig.3 for a LBBB case (depolarization wave starting on the right ventricle) after registration and interpolation of the Ensite non contact mapping data available only on the left ventricle.

\subsection{Mechanical Personalization Strategy}

In this paper, a SOFA ${ }^{4}$ implementation of the Bestel-Clément-Sorine electromechanical model (Marchesseau et al, 2012a,b), first introduced by Bestel et al (2001), was used (see Appendix A for a description of the model and its parameters). Using SOFA allows efficient computation, modularity and interactivity which are strong assets for further therapy planning. This model includes several passive and active terms, as well as some boundary constraints that

${ }^{4}$ SOFA is an open source medical simulation software (available at www.sofa-framework.org) 


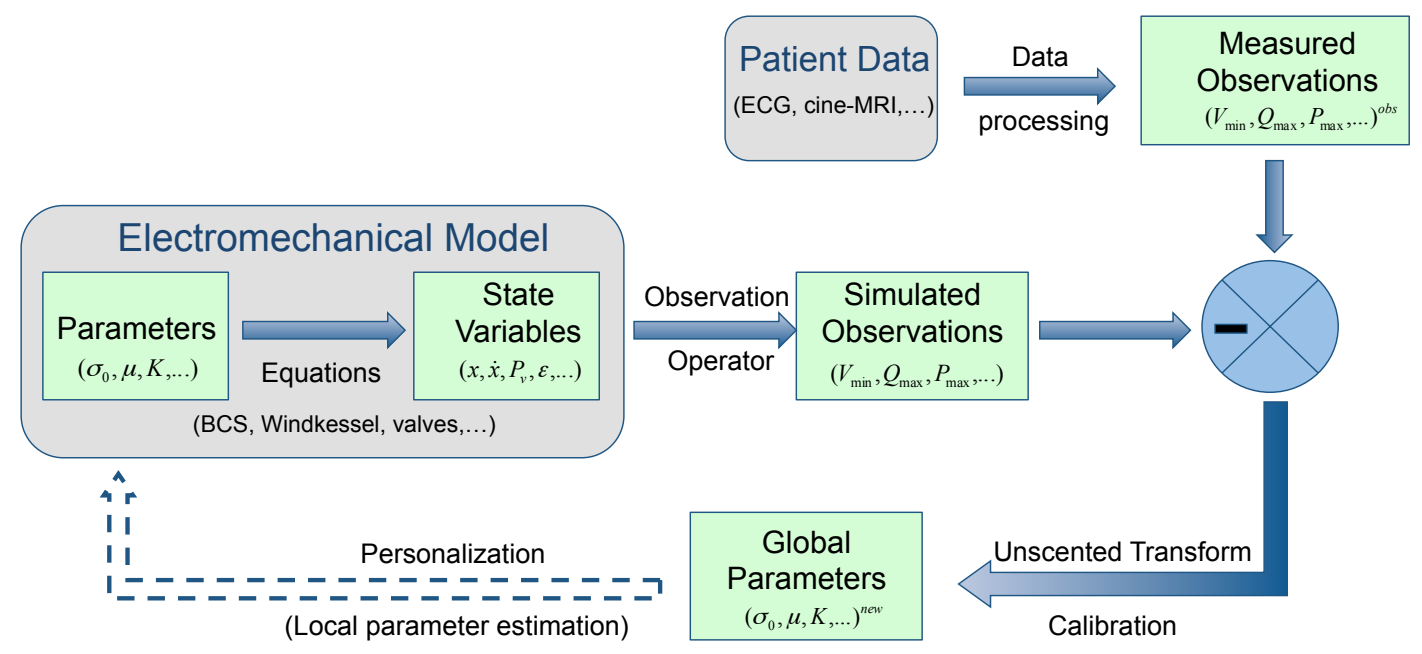

Fig. 4: Personalization pipeline: combining the electromechanical model and the data to calibrate then personalize the parameters.

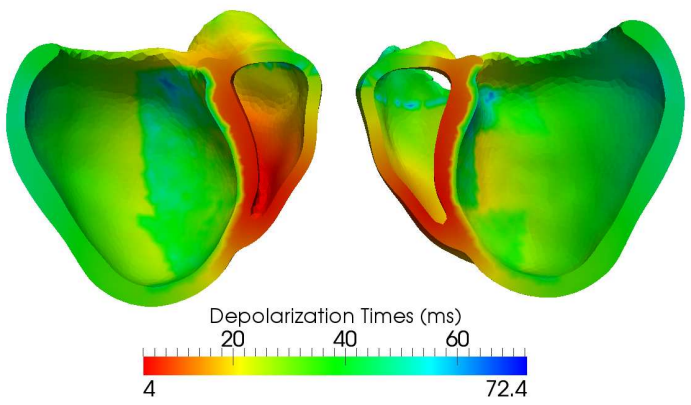

Fig. 3: Personalized electrophysiology simulation obtained after registration and interpolation of the EnSite Depolarization Time isochrones available on the Left Ventricle. (For interpretation of the color map in this figure, the reader is referred to the web version of this article.)

represent the fluid flow that circulates inside the ventricles during the four cardiac phases (Marchesseau et al, 2012a). This model depends on 14 global parameters that need to be estimated. To this end, we propose to first assess global values for the parameters using the Unscented Transform algorithm (Julier and Uhlmann, 1997) from global observed quantities (endocardial volume and pressure) as described in (Marchesseau et al, 2012b); and then to use the ROUKF (Reduced Order Unscented Kalman Filter) personalization algorithm (Moireau and Chapelle, 2011) implemented in Verdandi (Chapelle et al, 2012a) from local measurements to assess more precisely the contractility per region (see Fig.4 for a representation of this pipeline).

\subsubsection{Calibration based on the Unscented Transform}

The initialization of the parameters is a crucial prerequisite for the personalization algorithm to converge towards a relevant solution. In (Marchesseau et al, 2012a), we showed that this can be performed in a fast and efficient way using the Unscented Transform algorithm to match indices on the volume and pressure curves (if available) leading therefore to the calibration of 4 to 7 parameters. This algorithm, represented in Fig.5, builds a covariance matrix $\operatorname{Cov}(\theta, \mathbf{Z})$ between the relevant parameters which are spread around some initial parameter set $\theta^{0}$ and the observations $\mathbf{Z}$-in our case the minimum of the LV (Left Ventricle) volume and the minimum and the maximum of its derivative. The new parameters $\theta^{\text {new }}$ are then found to minimize

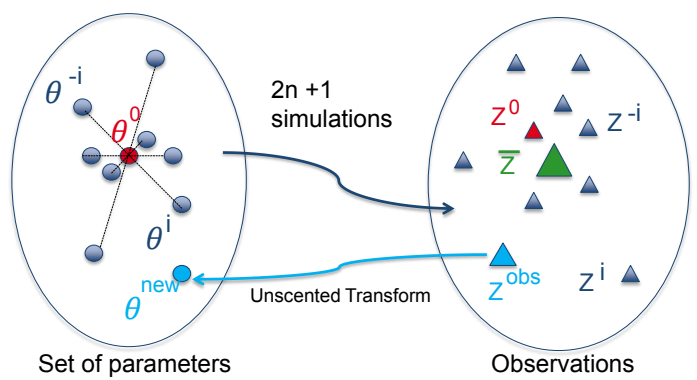

Fig. 5: Schematic representation of the Unscented Transform algorithm.

the difference between the mean simulated observations $\overline{\mathbf{Z}}$ and the measured observations $\mathbf{Z}^{\text {obs }}$ with

$\left(\theta^{\text {new }}-\theta^{0}\right)=\operatorname{Cov}(\theta, \mathbf{Z}) \operatorname{Cov}(\mathbf{Z}, \mathbf{Z})^{-1}\left(\mathbf{Z}^{o b s}-\overline{\mathbf{Z}}\right)$.

In one simulation, this algorithm enables to calibrate the main parameters chosen from a sensitivity analysis on the volume curve (and the pressure curve 
when available). We adjusted the initial set $\theta^{0}$ after a trial and error approach on a healthy volunteer and then used the same initialization $\theta^{0}$ for all studied cases (healthy and heart failure). Using the same $\theta^{0}$ and range of parameter values for all cases makes the calibration automatic. In this respect, we have already presented consistent results for healthy controls as well as pathological cases in (Marchesseau et al, 2012c).

\subsubsection{ROUKF Personalization Algorithm}

Once initialized, the parameters can be locally (regionally) estimated using a more advanced algorithm that deals with much larger dimensional systems. We use in this paper a type of Kalman filter which belongs to the sequential data assimilation methods.

\section{Data Assimilation Principles}

Data assimilation methods intend to estimate state variables of the considered system using measurements. State variables usually consist of the trajectory (positions, velocities ,...) but can in fact include model parameters or other dynamical variables such as regional strains for instance. A model is therefore represented by a dynamical system governing the state variable $\mathbf{X}$ that includes the parameters of the model:

$$
\left\{\begin{array}{l}
\dot{\mathbf{X}}=\mathscr{A}(\mathbf{X}, t) \\
\mathbf{X}(0)=\mathbf{X}_{0}+\xi_{X}
\end{array}\right.
$$

where $\mathscr{A}$ is a non-linear operator, $\mathbf{X}_{0}$ the a priori value for initial conditions and $\xi_{X}$ the uncertainties in the initial conditions.

The available measurements are described through a linear observation operator $\mathbf{H}$. The observations are therefore modeled by the application of this operator:

$$
\mathbf{Z}=\mathbf{H X}+\chi
$$

where $\chi$ is the observation noise.

Two types of assimilation methods exist: the sequential and the variational approaches. In the variational approach, a cost function is iteratively minimized after gathering measurements over a period of time. In the sequential approach, a new estimation of the state is performed each time a new measurement is available through statistical analysis. Both approaches have shown promising results in cardiac personalization: (Billet, 2010; Delingette et al, 2012; Sainte-Marie et al, 2006) that use the variational approach to estimate the contractility parameters or (Xi et al, 2011; Chabiniok et al, 2011) that preferred the sequential approach). We use in this paper the Unscented Kalman Filter in its reduced form, derived in (Moireau and
Chapelle, 2011) since we only estimate the parameters.

\section{Unscented Kalman Filtering}

The Unscented Kalman Filter can be seen as an iterative Unscented Transform described in 2.2.1. It is based on three steps at each discrete time $n$, for the state vector $\mathbf{X}$ which consists in the state values (positions, velocities, ...) and the parameters $\theta$.

- Sampling: Creates the sigma-points (minimal set of sample points around the mean) given an interpolation rule leading to $\mathbf{I}$, using a state error covariance matrix $\mathbf{P}$ :

$\hat{\mathbf{X}}_{n}^{(i)+}=\hat{\mathbf{X}}_{n}^{+}+\sqrt{\mathbf{P}_{n}^{+}} \mathbf{I}^{(i)}$

were $\mathbf{X}^{(i)}$ is the $i$ th sigma-points associated with vector $\mathbf{X}$.

- Prediction: Estimates the next state as the mean of the states simulated with the sigma-points, and the next covariance as the covariance of the states simulated with the sigma-points:

$$
\left\{\begin{array}{l}
\hat{\mathbf{X}}_{n+1}^{-}=E_{\alpha}\left(\mathbf{A}_{n+1 \mid n}\left(\hat{\mathbf{X}}_{n}^{*+}\right)\right) \\
\mathbf{P}_{n+1}^{-}=\operatorname{Cov}_{\alpha}\left(\mathbf{A}_{n+1 \mid n}\left(\hat{\mathbf{X}}_{n}^{*+}\right)\right)
\end{array}\right.
$$

where the mean $E_{\alpha}$ and the covariance $\operatorname{Cov}_{\alpha}$ are calculated with the weights $\alpha_{i}$ of the sigma-points, and noting $\mathbf{X}^{*}$ the concatenation of the vectors $\mathbf{X}^{(i)}$.

- Correction: Updates the state vector and its covariance given the new sigma-points and corresponding observations using the new observation operator $\mathbf{H}$ and the covariance of the noise $\mathbf{W}$ through the following calculations:

$$
\left\{\begin{array}{l}
\hat{\mathbf{X}}_{n+1}^{(i)-}=\hat{\mathbf{X}}_{n+1}^{-}+\sqrt{\mathbf{P}_{n+1}^{-}} \mathbf{I}^{(i)} \\
\mathbf{Z}_{n+1}^{(i)}=\mathbf{H}_{n+1} \hat{\mathbf{X}}_{n+1}^{(i)-} \\
\mathbf{P}_{\alpha}^{X \tilde{Z}}=\operatorname{Cov}_{\alpha}\left(\mathbf{X}_{n+1}^{*-}, \mathbf{Z}_{n+1}^{*}\right) \\
\mathbf{P}_{\alpha}^{\tilde{Z}}=\mathbf{W}_{n+1}+\operatorname{Cov}_{\alpha}\left(\mathbf{Z}_{n+1}^{*}, \mathbf{Z}_{n+1}^{*}\right) \\
\hat{\mathbf{X}}_{n+1}^{+}=\hat{\mathbf{X}}_{n}^{-}+\mathbf{P}_{\alpha}^{X \tilde{Z}}\left(\mathbf{P}_{\alpha}^{\tilde{Z}}\right)^{-1}\left(\mathbf{Z}_{n+1}-E_{\alpha}\left(\mathbf{Z}_{n+1}^{*}\right)\right) \\
\mathbf{P}_{n+1}^{+}=\mathbf{P}_{n+1}^{-}-\mathbf{P}_{\alpha}^{X \tilde{Z}}\left(\mathbf{P}_{\alpha}^{\tilde{Z}}\right)^{-1}\left(\mathbf{P}_{\alpha}^{X \tilde{Z}}\right)^{T}
\end{array}\right.
$$

\section{Reduced Order Unscented Kalman Filtering}

We aim, in this paper, at estimating only the $p$ parameters of the model (in our case the 17 regional contractilities from the 17 AHA zones of the left ventricle). Therefore, we only generate $2 p+1$ sigma-points and the covariance $\mathbf{P}$ is reduced to the parametric space of dimension $p$. Its rank is therefore $p$, much smaller that the dimension of the space $d$. We suppose that we can manipulate covariance matrices in the factorized form:

$\mathbf{P}=\mathbf{L} \mathbf{U}^{-1} \mathbf{L}^{T}$ 
where $\mathbf{U}$ is an invertible matrix of rank $p$ and represents the main uncertainties of the system. A new derivation of the algorithm can therefore be performed without computing the full covariance matrix $\mathbf{P}$, as shown in (Moireau and Chapelle, 2011; Moireau et al, 2008).

\subsubsection{Observation Extraction from the Images}

We suppose in this paper that the deformed meshes registered from the cine-MRI as explained in Sec.2.1.2 are the only available measurements. Several types of observations can then be extracted from these meshes. Since the registration method focuses only on the visible contours in the image, we cannot expect the trajectory of all the points in the myocardium to be accurate. We will therefore only consider the points on the endocardial surfaces. Three types of observations can be derived directly from this:

\section{Volume curve}

First, for the calibration, the volume of the Left Ventricle is computed over time, giving the three required observations $\left(V_{\text {min }}, d V / d t_{\min }\right.$ and $\left.d V / d t_{\max }\right)$. These observations are easily computed using the topology of the endocardium as explained in (Marchesseau et al, 2012a). However it is not sufficient to recover the motion of the whole myocardium.

\section{Positions on the Surface}

The most complete observation include the positions of all the points on the endocardial surface. The estimation tries therefore to match the motion point-wise. The advantage of what we call the Surface-Points Estimation is that the observation operator $\mathbf{H}$ is straightforward, filled with 1 values on each coordinate of the surface nodes and 0 values otherwise. The main drawback is that there is no guarantee that the registration tracks displacements of material points, but rather apparent displacements of the contours, inducing therefore errors on the observations (well known aperture problem in Computer Vision where several deformations would lead to the same visible contours). There exist observation operators Chabiniok et al (2011) that filter this aperture issue taking into account distances between the surface contours. These methods give promising results but are not directly applicable for clinical context due to their computation times.

\section{Regional Volumes}

Alternatively, we suggest to use regional volumes as observations. To this end, we project the 17 AHA volumetric segments (Fig.6) on the LV endocardium surface, and calculate the volume formed by this surface and the barycenter of the LV endocardium over time. Not only does this method allow to capture the motion of the surface locally without smoothing tracking errors, but it also leads to a small number of observations to match, decreasing this way the computation time. Note that a possible drawback could be a loss of observability (or identifiability here) due to the reduction of the observation space. However, regional volumes are used by clinicians as a pathological index (Baxley and Joseph Reeves, 1971) and therefore may contain sufficient information on the investigated pathology.

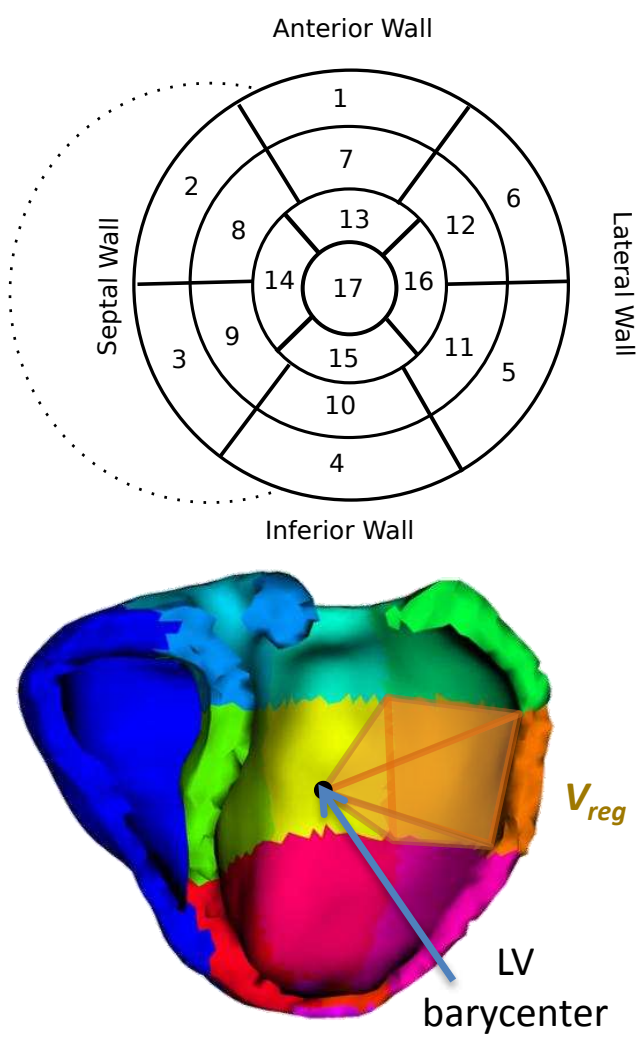

Fig. 6: (Top) Bull's eye plots of the AHA 17 segment division (View from the apex). (Bottom) Example of Regional Volume.

\subsubsection{Error Measurements}

In order to evaluate the errors between the estimated motion from the registration and the simulated motion, several error measurements are used. First, the result of the calibration algorithm compares the simulated volume curve $V^{\text {simu }}(t)$ to the registered volume curve $V^{o b s}(t)$ using the standard root mean square difference:

$\bar{\varepsilon}_{V}=\sqrt{\frac{\sum_{t=0}^{N_{\text {steps }}}\left(V^{\text {simu }}(t)-V^{\text {obs }}(t)\right)^{2}}{N_{\text {steps }}}}$ 
where $N_{\text {steps }}$ is the number of images in the MRI sequence. Then, after personalizing the model from regional volumes, we compare the simulated regional volumes $V_{i}^{\text {simu }}(t)$ to the registered ones $V_{i}^{\text {obs }}(t)$ normalized by the area $S_{i}$ of the AHA surface (for an estimation of the distance between the surfaces) to obtain a mean square difference for each regional volumes that we average:

$\bar{\varepsilon}_{\text {RegVol }}=\frac{1}{N} \sum_{i=0}^{N} \frac{1}{S_{i}} \sqrt{\frac{\sum_{t=0}^{N_{\text {steps }}}\left(V_{i}^{\text {simu }}(t)-V_{i}^{\text {obs }}(t)\right)^{2}}{N_{\text {steps }}}}$.

It is also interesting to record the distance error between the regional volumes at end-systole where it is maximal:

$E_{\text {RegVol }}=\frac{1}{N} \sum_{i=0}^{N} \frac{\left|V_{i}^{\text {simu }}\left(T_{\max }\right)-V_{i}^{\text {obs }}\left(T_{\max }\right)\right|}{S_{i}}$.

These errors give a good indication on how well the simulated motion match the registered one on the left endocardium. For a more global index that takes all the surfaces into account (including epicardium and right endocardium), we define a symmetric distance on the contours $\Omega$ as:

$$
\begin{aligned}
\varepsilon_{C}= & \frac{1}{2} \frac{\sum_{k=0}^{N_{\text {points }}} \operatorname{dist}\left(\Omega^{\text {simu }}-\mathbf{P}_{k}^{\text {obs }}\right)}{N_{\text {points }}} \\
& +\frac{1}{2} \frac{\sum_{k=0}^{N_{\text {points }}} \operatorname{dist}\left(\Omega^{\text {obs }}-\mathbf{P}_{k}^{\text {simu }}\right)}{N_{\text {points }}}
\end{aligned}
$$

with a mean value over time noted $\bar{\varepsilon}_{C}$ and a value at end-diastole $E_{C}$.

\section{Results}

\subsection{Selection of Adequate Observations}

In order to select the most appropriate type of observations (surface points vs LV endocardium regional volumes), we performed a synthetic study. We created a sequence of deformed meshes with a forward simulation of the model using different values of the contractility for each of the 6 zones of the left ventricle (combining the AHA segments as shown in Fig.7). We then estimated these 6 contractilities with the ROUKF personalization algorithm using either the surface points as observations or the regional volumes.

The resulting deformed meshes after personalization are compared with the ground truth (simulated data) on Fig. 8 where we can see that both methods give an excellent match. The resulting estimated parameters are given in Fig.9 for the two estimations

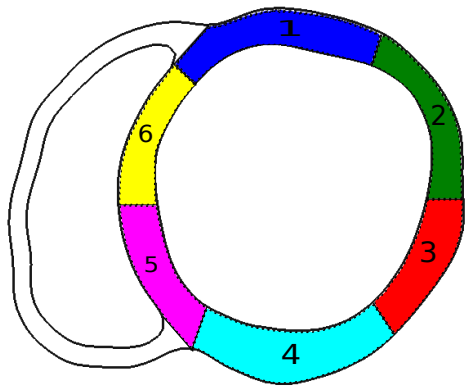

Fig. 7: Division of the myocardium into 6 regions for the left ventricle and 1 for the right ventricle, combining the standard AHA zones. (View from apex)
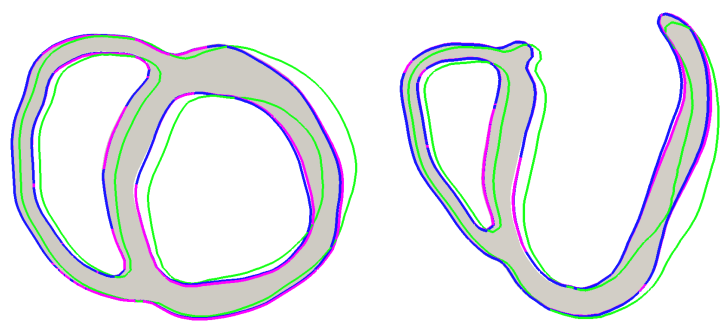

Fig. 8: Comparison of the personalized motion at end-systole on short axis view from the base (left) and long axis (right) plane. Grey surfaces represent the ground truth, green contours the initial simulation, pink the estimated contours using regional volumes and blue the estimated contours using surface points. Since no clear difference can be made from the pink and blue contours, we conclude that both techniques give similar results and manage to match the ground truth. (For interpretation of the references to color in this figure legend, the reader is referred to the web version of this article.)

and compared with the reference value. We can see that surprisingly the parameters are better estimated with the regional volumes. To understand why observing regional volumes leads to better parameters in this case than observing all the points of the surface, we evaluated the errors on these two types of observations (Surface Point error $\bar{\varepsilon}_{C}$ and Regional Volumes error $\bar{\varepsilon}_{\text {RegVol}}$ ) while varying the contractility of one zone around its ground truth value. Fig. 10 gives the resulting mean errors. We can see that the Regional Volumes error $\bar{\varepsilon}_{\text {RegVol }}$ has only one minimum whereas the Surface Points error $\bar{\varepsilon}_{C}$ has a non-smooth variation leading to several local minima in which the estimated parameters may be "trapped" in the filtering process. Of course, observability is enhanced when using pointwise displacements, hence we conjecture that a finer tuning of the filtering method would overcome this difficulty, see Imperiale et al (2011). 

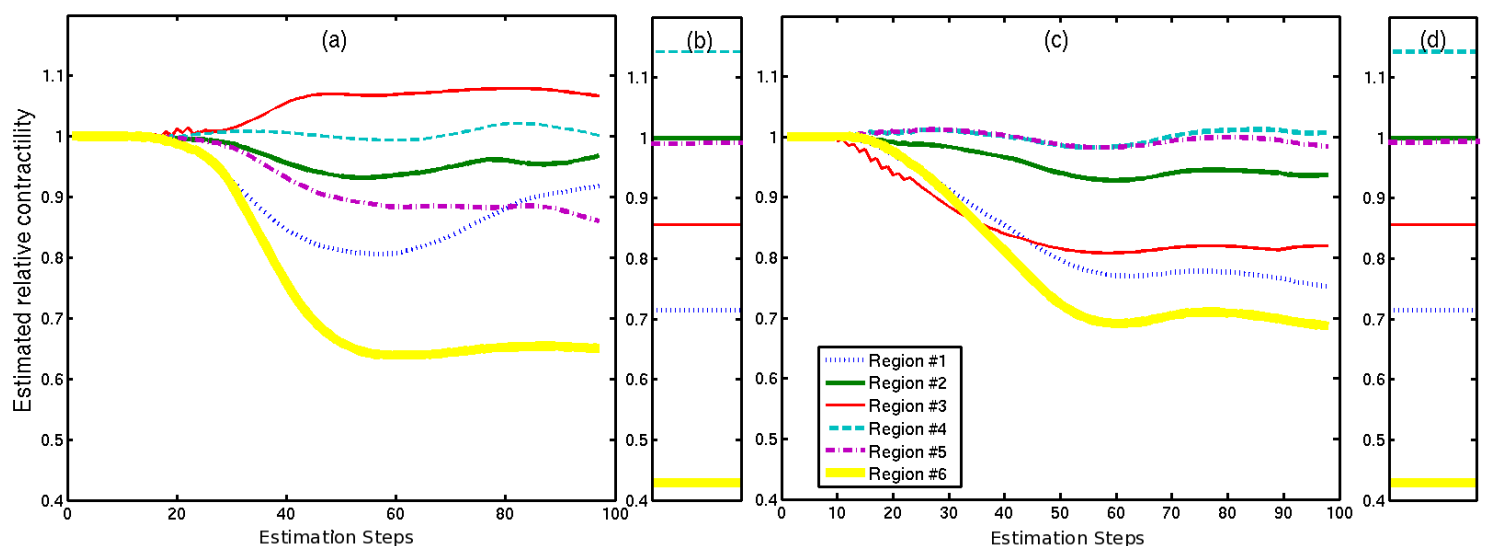

Fig. 9: (a) Regional contractility estimation using observations on all the surface points. (b)(d) Reference values of the contractility. (c) Regional contractility estimation using observations on the regional volumes in the LV endocardium.

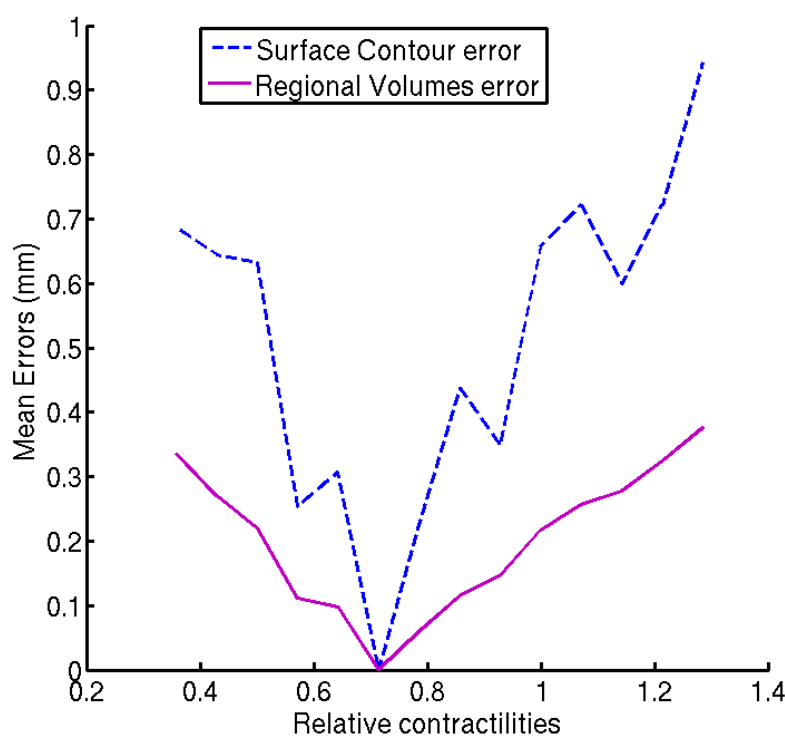

Fig. 10: Comparison of the errors using the two types of observations, for various contractilities.

\subsection{Detection of Infarcted Tissue}

In this section, we tested the ability of a personalized model, using the regional volumes as observations, to detect regions of low contractility, for instance due to presence of scars following an infarct. In order to evaluate the detection power of the proposed personalization based on regional volumes, a set of tests were performed using synthetic data. Forward simulations with varying size of scar tissue (from 300 tetrahedra to 2000 tetrahedra out of the 65000 tetrahedra for the full myocardium) were performed to create the observations. The relative contractility was set to 0.3 for the scar zone and 1 for the rest of the myocardium. Before using the personalization algorithm, random noise was added on the regional volumes to obtain obser- vations closer to what would be observed with real data. Resulting volumes curves (with $10 \%$ noise) compared to synthetic ones (0\% noise) are shown in Fig. 11. Moreover, to calibrate the ROUKF personalization algorithm, the expected maximum error must be set. To make this test realistic, an error of $0.5 \mathrm{~mm}$ was allowed. This explains why the initial relative contractility of 0.3 cannot be reached.

The estimated contractilities obtained for the scars

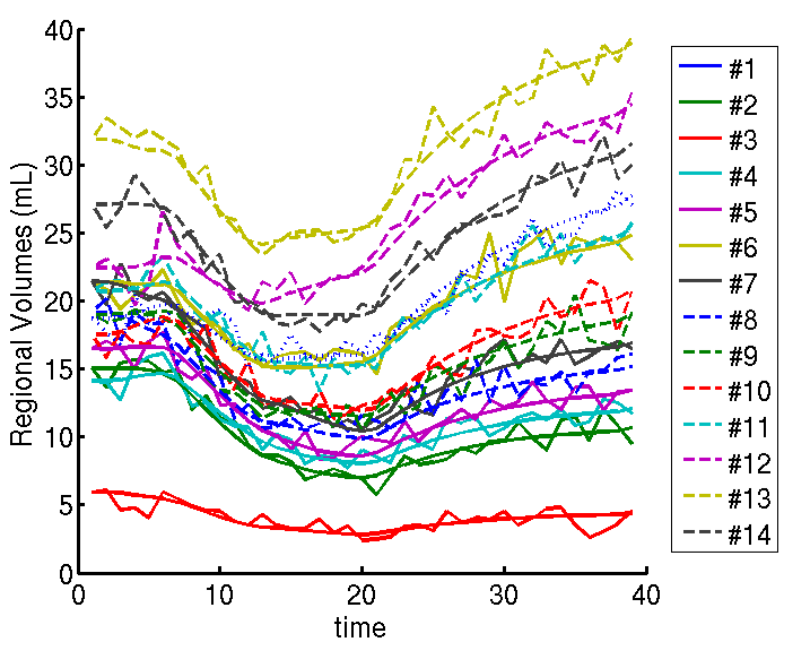

Fig. 11: Regional Volumes simulated with $0 \%$ noise (smooth curves) and $10 \%$ noise (non-smooth curves).

zone are presented Fig.12 for the various sizes and noise amplitudes. We note that the noise does not influence the estimation of the parameter for scars zone larger than 1300 tetrahedra (as represented in Fig.12). Moreover, since a relative contractility of 1 represents the normal contractility for the myocardium, an estimated relative contractility too close to 1 for the scar zone means that the detection fails (which is the case 
for the zones with sizes 300 tetrahedra, 650 tetrahedra and 1300 tetrahedra). On the other hand, the zone of size 2000 tetrahedra has an estimated relative contractility much smaller than 1 which means that the detection succeeds. Therefore, the proposed personalization method based on regional volumes is able to detect zones with lower contractility as long as these zones are large enough (here $3 \%$ of the myocardium).

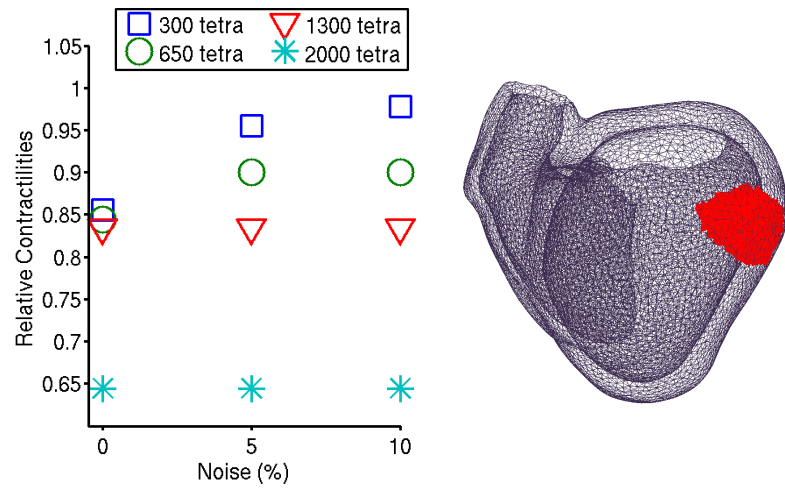

Fig. 12: (Left) Estimated contractilities on the scars zones for different sizes of the zones and different noise. (Right) Representation of a scar zone of 1300 tetrahedra.

\subsection{Real Cases Personalization}

Personalizing real cases requires three steps:

- Selecting the region that has the smallest displacement (basal area or apex) in order to constraint this region as a boundary condition.

- Performing a calibration of 4 parameters (including contractility) for the left ventricle, based on the volume curve.

- Running the ROUKF personalization algorithm using regional volumes on the LV endocardium in order to estimate the 17 regional contractilities of the LV.

This pipeline was applied on 3 pathological cases and 8 healthy volunteers and enabled to draw preliminary conclusions on pathological cases versus healthy controls. Results of the full personalization strategy are detailed here for the pathological Case \#1, then error measurements and estimated contractilities are given on all cases. The observation vector $\mathbf{Z}$ is of size the number of regional volumes and $\mathbf{X}$ contains all the state variables and the parameters to estimate (node positions, velocities, ...). The full pipeline takes about $2 p+2=36$ times a simulation time on a single CPU (one simulation lasts about 10 minutes for 1 heart beat on a 60000 tetrahedral mesh) where $p$ is the number of estimated parameters (here 17 regional contractilities). However, full parallelization can be done leading to a total computation time of twice a simulation time (20 minutes).

\subsubsection{Results for Case \#1}

First, volume curves resulting from the calibration are given in Fig.13. The mean error on the volume $\bar{\varepsilon}_{V}$ decreases from $17 \mathrm{~mL}$ to $4 \mathrm{~mL}$ giving therefore a good initialization.

Second, the results of the full personalization are given

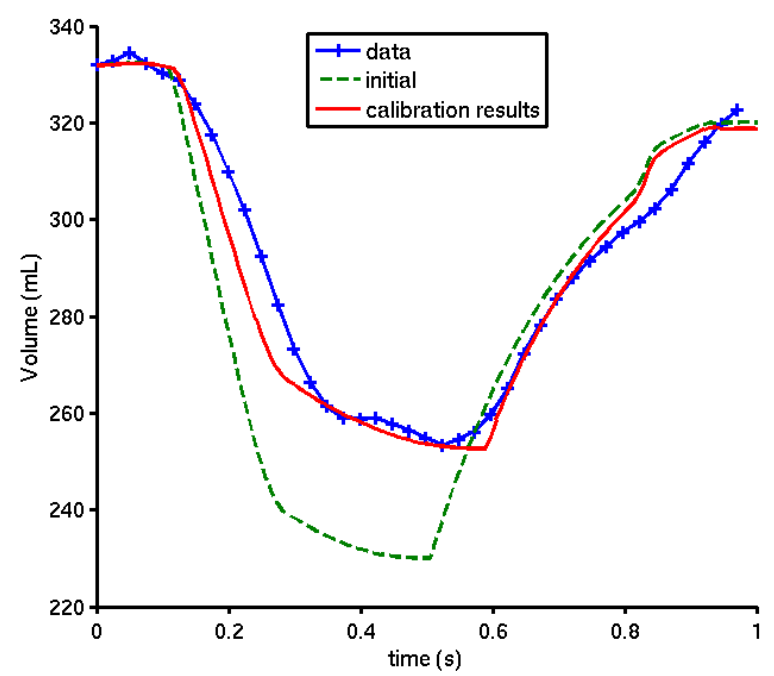

Fig. 13: Case \#1: Volume evolution before (green) and after (red) calibration compared with real value extracted from the registration (blue)

in Fig. 14 where we compare the deformed mesh to the registered mesh and the images. The distance error on the contours $\varepsilon_{C}$ is displayed in Fig.15 at end-diastole and shows how close the model follows the registered motion. The mean distance error on all the surface decreases from $2.8 \mathrm{~mm}$ to $2.1 \mathrm{~mm}$ which is a reasonable error given the image resolution $(1.25 \mathrm{~mm} \times 1.25 \mathrm{~mm})$ and the fact that we only try to match the left endocardium.

Registered and simulated regional volume curves are compared in Fig.16. The mean relative error on the regional volumes (normalized by the surface area) decreases from $\bar{\varepsilon}_{R e g V o l}=1.1 \mathrm{~mm}$ with initial parameters to $\bar{\varepsilon}_{\text {RegVol }}=0.6 \mathrm{~mm}$ after personalization which proves a very good improvement.

Finally, Fig. 17 gives the estimated relative contractilities along the personalization steps and shows that the 

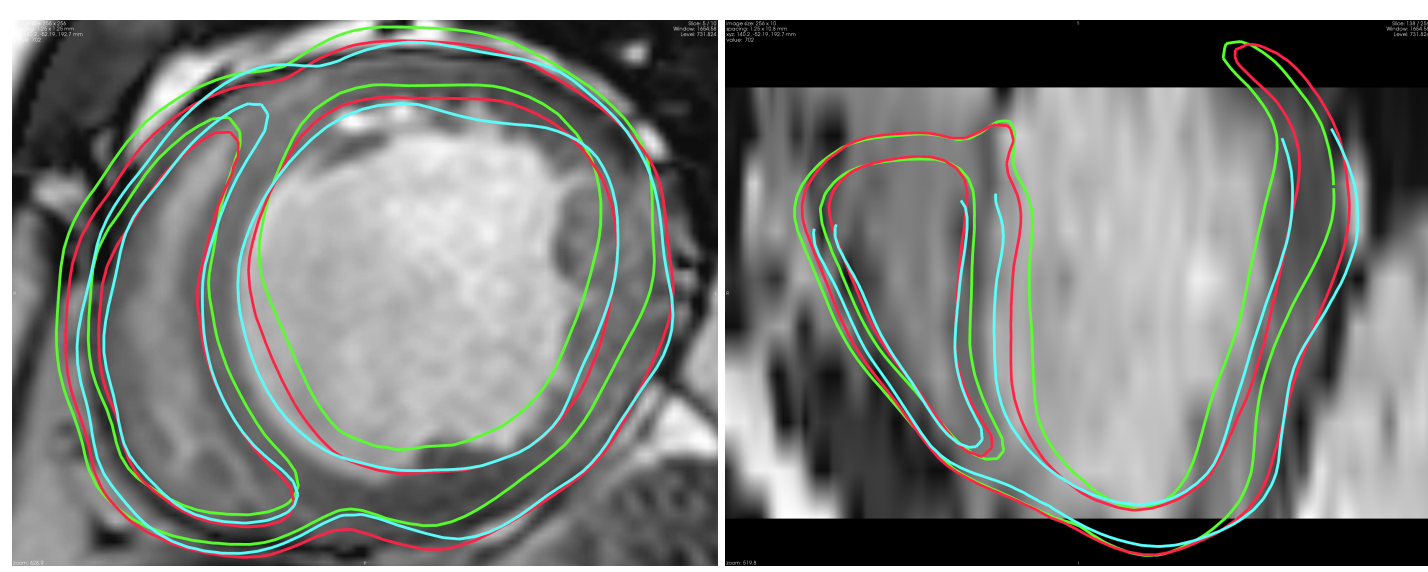

Fig. 14: Short axis and long axis view of the end-systolic phase. Comparison of direct model with the initial parameters (green), direct model with the estimated contractilities on all left ventricle AHA zones using the regional volumes on the LV endocardium (red) and registered mesh (cyan). (For interpretation of the references to color in this figure legend, the reader is referred to the web version of this article.)
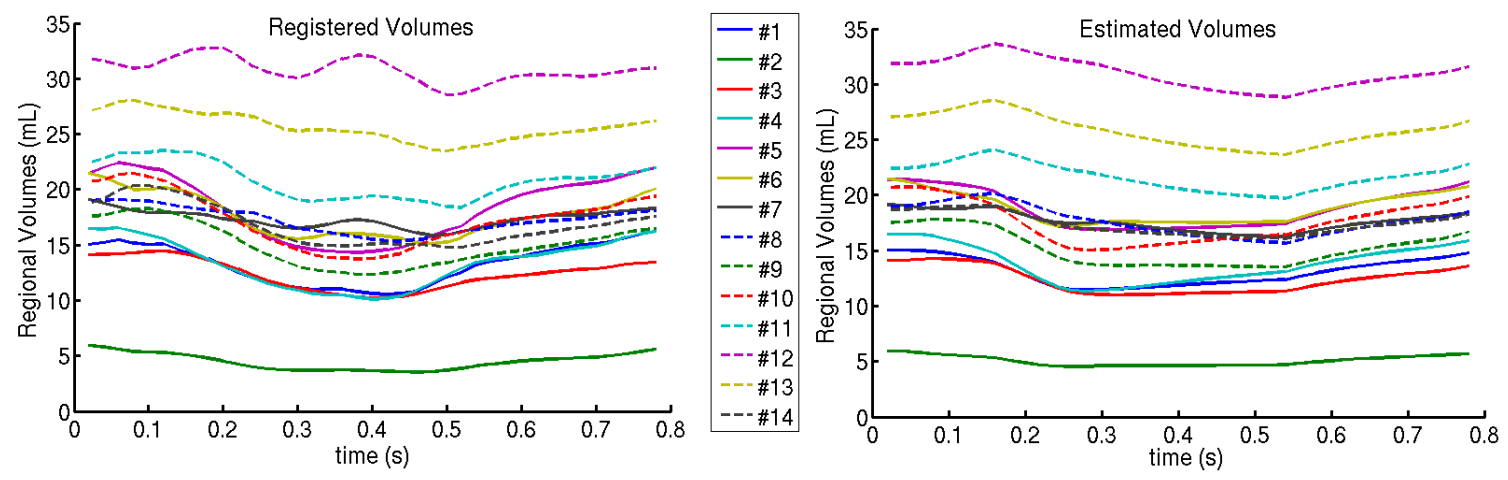

Fig. 16: Case \#1: (Left) Registered regional volumes on the LV endocardium. (Right) Estimated regional volumes.

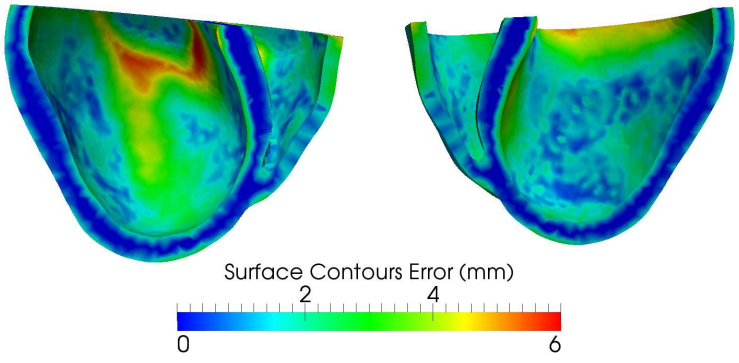

Fig. 15: Case \#1: Evaluation of the distance error on the contours of the surface at end-systolic phase $\left(E_{C}\right)$. The basal area is cropped since we do not take the corresponding observations into account. (For interpretation of the color map in this figure, the reader is referred to the web version of this article.)

estimation converges before the end of the personalization.

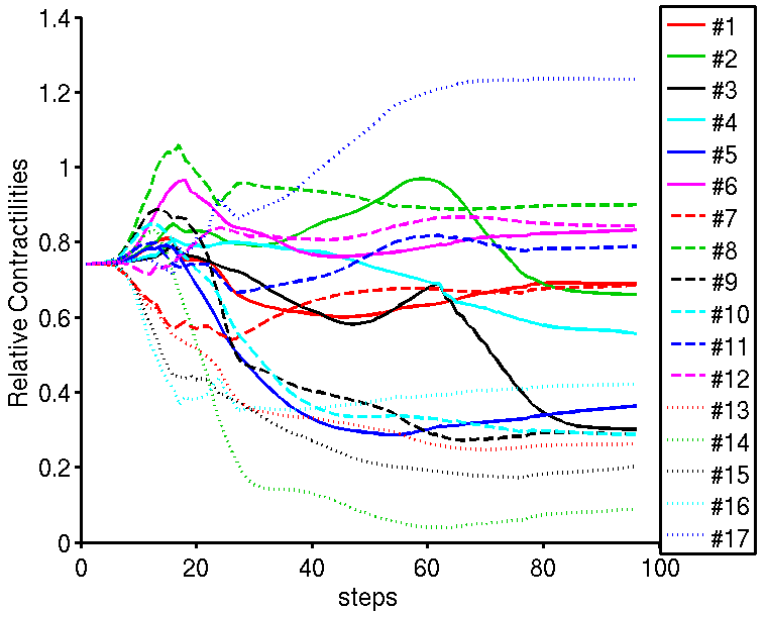

Fig. 17: Case \#1: Evolution of the estimated relative contractilities along the personalization steps. We note a mean relative contractility of 0.55 . Contractility of zone \#17 might be overestimated since it corresponds to the apex which is constrained by our boundary conditions. 


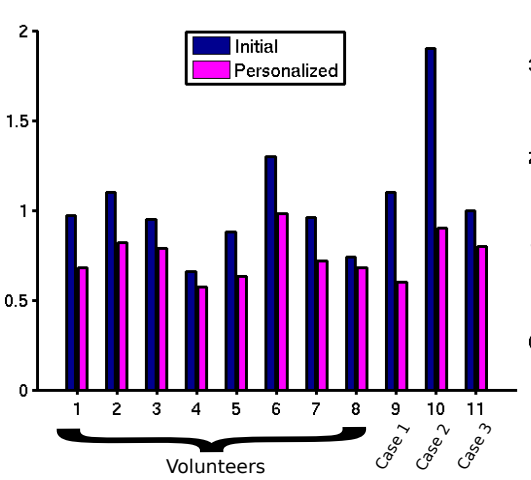

(a) $\bar{\varepsilon}_{\text {RegVol }}(\mathrm{mm})$

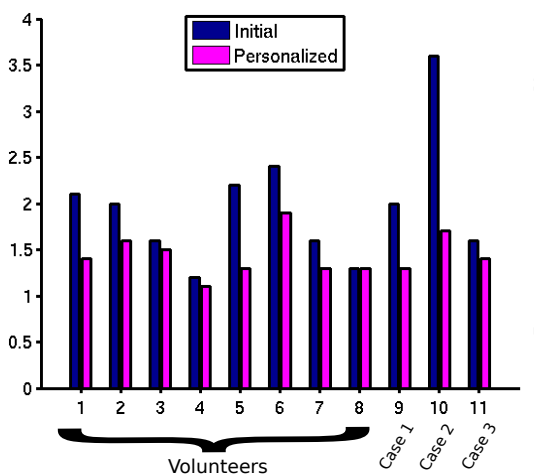

(b) $E_{\text {RegVol }}(\mathrm{mm})$

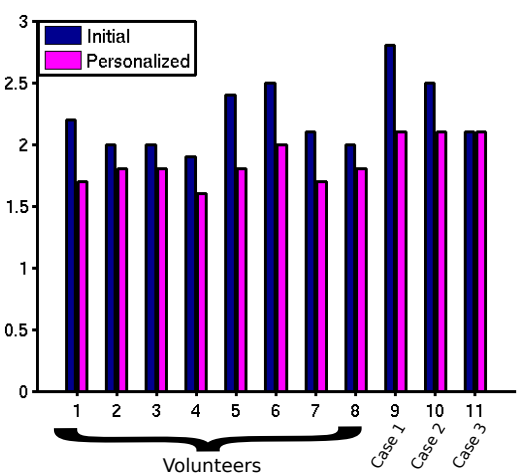

(c) $\bar{\varepsilon}_{C}(\mathrm{~mm})$

Fig. 18: Errors between the simulation and the registration before (in blue) and after (in pink) personalization.

\subsubsection{Error Measurements}

In order to evaluate the accuracy of the personalized motion, we computed the errors previously defined on all the tested cases, before and after personalization. Fig.18 presents in (a) the resulting errors on the regional volumes $\bar{\varepsilon}_{\text {RegVol }}$ which is what is actually minimized during the personalization, as well as its value at end-systole (b). Finally, as an indicator of the global motion on all the surfaces, the distance error on the contours $\bar{\varepsilon}_{C}$ is given in (c).

We can see that the personalization decreases the errors in all the cases and that the mean error on the regional volumes is pretty low $(0.7 \mathrm{~mm})$ which is smaller than a pixel size. However, the error on the total surface still needs to be improved including observations from the right ventricle and the epicardium.

\subsection{Preliminary Specificity Study}

Our database consisting of 8 healthy controls and 3 pathological cases selected for the Cardiac Resynchronization Therapy (with therefore a small ejection fraction and a long QRS) allows to draw preliminary conclusions and compare each pathological case to the controls. First, from the global volume curves, a calibration was performed to initialize 4 parameters. The resulting parameters for the healthy controls (box plots) and each pathological case are presented in Fig.19. We can see from this graph that the Computational Biophysical Model Personalized for case 1 (CBMP\#1) and case 2 (CBMP\#2) exhibit a similar behavior with a smaller global contractility than the healthy controls but normal bulk modulus, viscosity and peripheral resistance. On the other hand, the Computational Biophysical Model Personalized for the third case (CBMP\#3) exhibits what seems to be a global contractility closer to the normal range, and a very high Bulk Modulus. Therefore, the model of the myocardium muscle for this case is stiffer than the model describing the healthy cases but the contractility is equivalent. The small ejection fraction can therefore be due for CBMP\#3 to the high stiffness whereas it can be due for CBMP\#1 and CBMP\#2 to a small contractility. This difference could be explained by the fact that this patient has a different etiology than the first two cases (idiopathic cardiomyopathy) and, more importantly, has regurgitation at both the mitral and aortic valves. Furthermore, this patient presents significant atrial volumes, with an increased pulmonary artery pressure. This leads to a smaller inflow than outflow as shown in Fig.20.

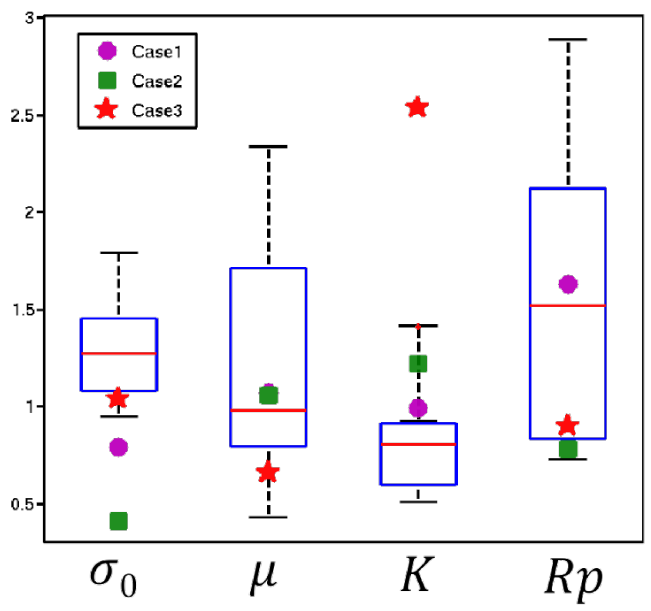

Fig. 19: Estimated global parameters for the 3 pathological cases compared to the healthy controls (box plots). $\sigma_{0}$ is the global contractility, $\mu$ the friction parameter, $K$ the Bulk modulus related to the stiffness of the passive myocardium and $R_{p}$ the peripheral resistance in the aorta. 


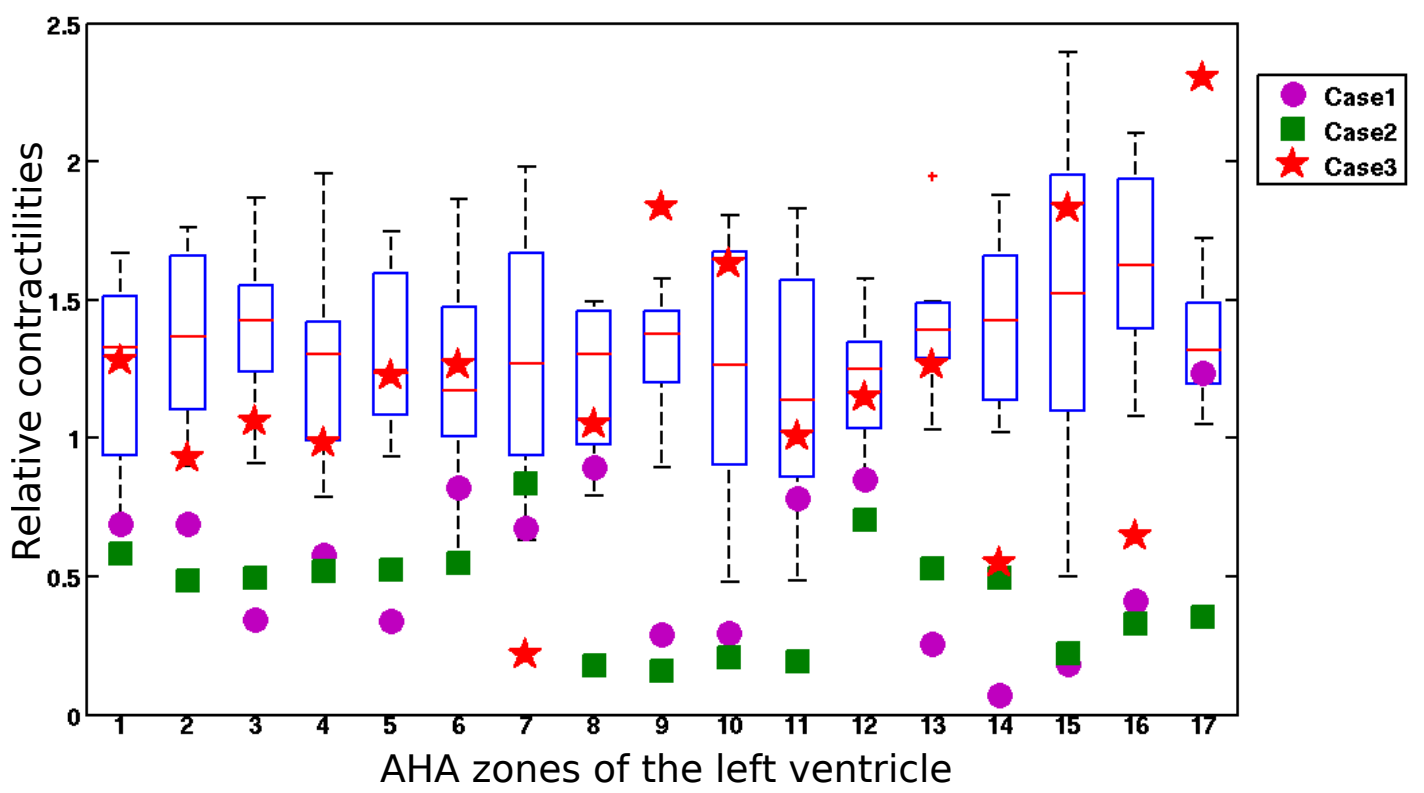

Fig. 21: Estimated regional contractilities for the 3 pathological cases compared to the healthy controls (box plots).

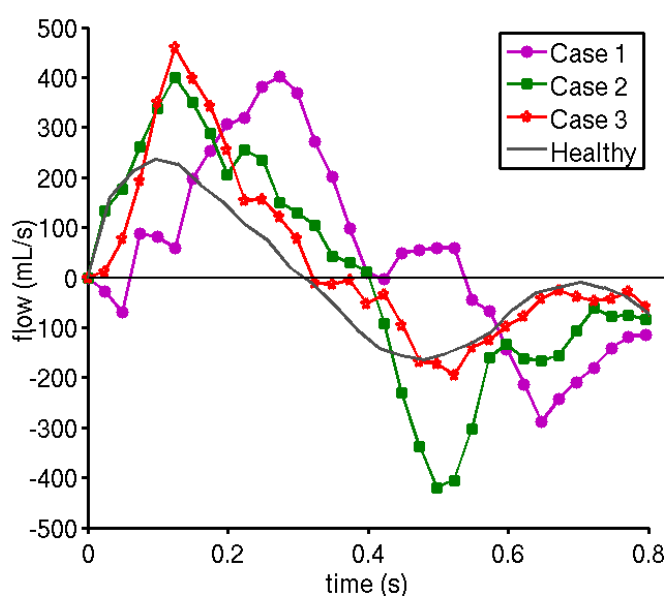

Fig. 20: Registered outward flow curves for the pathological cases compared to the mean healthy curve, showing that Case 3 only has a smaller inflow than outflow.

A comparison of the regional contractilities obtained from the personalization method that we propose leads to consistent observations (see Fig.21). Indeed, the personalized regional contractilities of the first two cases are much smaller on all the regions than the controls. Moreover, CBMP\#3 exhibits regional contractilities closer to the contractilities of healthy hearts for most regions. Some of the estimated contractilities however are extreme (zone 7 has a very small contractility while 17 has a very high contractility) which could indicate local dysfunctions. The study of the covariance on the parameters showed that our con- fidence on the contractilities of zones 1 to 6 and zone 17 is 4 times smaller than on the other zones due to the boundary constraints on the base and the apex that we set.

\section{Discussion}

\subsection{Personalization from Regional Volumes}

In this paper we proposed patient-specific modelling of the heart based on regional volumes. Tests performed on synthetic data proved that this choice of observations was relevant to estimate regional contractilities and detect scar tissue as long as the size of the scar zone is not too small. The advantage of such an approach is to smooth the input observations in order to match the motion of the left endocardium. This method proved to be robust and can be used at a large scale. Indeed, although the regional volumes were rather different for pathological cases and healthy volunteers, we managed to estimate the regional contractilities and have a good final match on the motion. Finally estimating the regional volumes gives precious indices on the synchronization of the AHA zones which could be further studied for therapy planning.

\subsection{Analysis of Real Case Personalizations}

Our database of 3 pathological cases and 8 volunteers allows to draw preliminary conclusions. First, the per- 
sonalized computational biophysical models that intend to characterize these pathological cases exhibit different global contractility and/or stiffness. Our results on the calibration and on the regional personalization are consistent and show that CBMP\#1 and CBMP\#2 display a similar behavior (small estimated global and regional contractilities with normal estimated stiffness), while CBMP\#3 is clearly different. First, CBMP\#3 exhibits a reasonable global contractility parameter while showing a high stiffness which seems to explain its small ejection fraction and its small filling flow. Second, CMBP\#3 estimated regional contractilities are for most of them similar to healthy cases with localized akinetic regions confirmed by the clinicians. In addition, this case, with a different etiology, was the one not responding to the CRT. Our personalization pipeline therefore gives results in agreement with the clinical interpretation of the images.

\subsection{Limitations of the Personalization Pipeline}

The proposed approach includes several processing and computational steps that are subject to uncertainties. First, we relied on image segmentation and tracking which are challenging due to the limited intensity contrast of the myocardium, and the aperture problem. Then, with the calibration, 4 global parameters could be assessed (contractility, viscosity, bulk modulus and peripheral resistance) and the remaining 10 (including other stiffness parameters, contraction and relaxation rates as well as other pressure related parameters) were set to their standard values which were chosen after a trial and error approach on one healthy case, or from the literature (see Appendix A for the full list and values). This choice of parameters to calibrate was made from a complete sensitivity analysis (Marchesseau et al, 2012a) which led to conclusive results on healthy and pathological cases (Marchesseau et al, 2012c). However, data on ventricular pressure would help the parameter estimation compared to using literature values that can be far from the actual patient condition. Second, boundary conditions play a significant role in the myocardium motion and hemodynamics, therefore they need to be assessed carefully. Our model includes a pericardium that constrains the outward radial motion and some elastic constraint that can be applied on the apex (which is either outside the field of view of the images, or barely moving) and on the basal area around the valves. However, the pre-load and the after-load were not personalized in this article which probably impacts the estimation of the parameters. For instance, modelling the regurgitation observed on the third pathological case could help in obtaining parameters closer to the actual intrinsic tissue properties of this patient. Finally, a time registration between the real data and the simulation is necessary and influences as well the results of the personalization.

\subsection{Perspectives}

Our method only intends to recover the motion of the left endocardium. To improve the results of the personalization, or estimate more parameters such as the regional stiffness for instance, one may include the regional volumes calculated from the epicardium and intend to recover the thickening and shrinking of the muscle or estimate the parameters related to the boundary conditions. Using measurements on the regurgitation would also improve the personalization since our heamodynamic model that represents the pressure constraint (Marchesseau et al, 2012a) can include regurgitation by estimating the iso factor $K_{i s o}$. Alternatively, we could couple this heamodynamic constraint with a lumped-flow model as used by (Koon et al, 2010). Also, since the estimated parameters indicate a highly incompressible behavior, we will address this incompressible behavior in the future by adopting specific finite element procedures. Moreover, we could divide the endocardium in different zones than the standard AHA zones in order to obtain more precise results (for instance taking into account known scar zone, or dividing the actual AHA zones by 2). Applying this method on a larger database could also lead to better assessment of the parameters specificity of the personalized models. Finally, the proposed method could also help in testing the acute response to pacing by simulating it on the personalized model.

\section{Conclusion}

This paper proposed a personalization strategy made of a global calibration and the application of the Reduced Order Unscented Kalman Filter to estimate regional contractilities based on regional volumes using a complete model of the heart. The personalization of 3 pathological cases and 8 volunteers shows the robustness of this strategy and opens up possibilities to study the specificity of the estimated physiological parameters to the etiology and the response to CRT. The use of regional volumes allows to smooth registration errors and to find a good compromise between the physiological behavior of the model and the accuracy of the personalization.

Acknowledgements This work was partially funded by the European Community's euHeart project under grant agreement 
224495 and by the ERC advanced Grant MedYMA 291080. The authors warmly thank Marc Fragu from INRIA, MACS team in Saclay, for his support and advice in using Verdandi, as well as Martha L.Aguilar from UPF whose help in processing the data was essential to this work and Michel Sorine from INRIA, SISYPHE team for constructive discussions.

\section{A The Bestel-Clément-Sorine electromechanical model}

For the sake of completeness, we provide a brief description of the Bestel-Clément-Sorine electromechanical model (Bestel et al, 2001) further improved by Chapelle et al (2012b) that we used throughout this paper. This description has already been published in (Marchesseau et al, 2012b).

The model is composed of a passive isotropic viscohyperelastic component that accounts for the elasticity and the friction in the cardiac extracellular matrix surrounding the fibres, described as a MooneyRivlin material. The strain energy for a MooneyRivlin material is given as:

$$
W_{e}=c_{1}\left(\bar{I}_{1}-3\right)+c_{2}\left(\bar{I}_{2}-3\right)+\frac{K}{2}(J-1)^{2}
$$

where $c_{1}, c_{2}$ are material parameters and $K$ is the Bulk modulus. The quantities $\bar{I}_{1}$ and $\bar{I}_{2}$ are the isochoric invariants of the Cauchy-deformation tensor $\mathbf{C}$.

In parallel, the stress along the cardiac fibre is composed of an active part (contraction in the sarcomere) and a passive part corresponding to the elastic bound (titin) between sarcomeres and Z-discs, having stress $\sigma_{s}=E_{s} e_{s}$. The contractile component having stress tensor $\sigma_{c}$, driven by the control variable $u$, has a viscous part to account for the energy dissipated in the sarcomere due to friction. This gives

$$
\sigma_{c}=\tau_{c}+\mu \dot{e}_{c}
$$

Fig. 22 shows a rheological representation of this model.

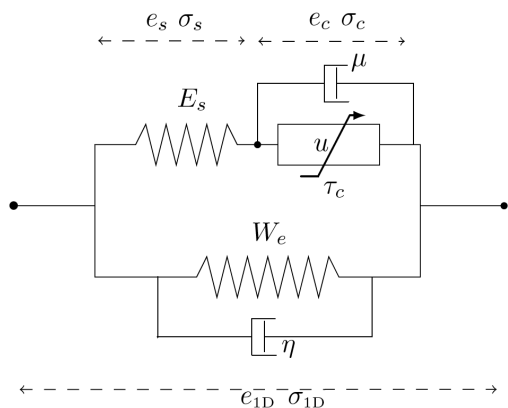

Fig. 22: Full electromechanical model. $W_{e}$ is the strain energy of the extracellular matrix considered here as an isotropic material, associated with a dissipative term $\eta . u$ is a control variable which is driven by changes in transmembrane potential. It controls the contraction stress $\tau_{c} . \mu$ deals with the friction in the sarcomere while $E_{s}$ is a linear spring to enforce elasticity of the Z-discs (titin).

At the nanoscopic scale, the binding and unbinding process of the actin and myosin filaments in the sarcomere is described by Huxley's filament model (Huxley, 1957). Statistical mechanics allows to describe its behavior at the macroscopic scale, resulting in a differential equation that controls the active stress $\tau_{c}$ and the sarcomere stiffness $k_{c}$ :

$$
\left\{\begin{array}{l}
\dot{k_{c}}=-\left(|u|+\alpha\left|\dot{e_{c}}\right|\right) k_{c}+n_{0} k_{0}|u|_{+} \\
\dot{\tau}_{c}=-\left(|u|+\alpha\left|\dot{e}_{c}\right|\right) \tau_{c}+\dot{e_{c}} k_{c}+n_{0} \sigma_{0}|u|_{+}
\end{array}\right.
$$

where $\alpha$ is a constant related to the cross-bridge release due to a high contraction rate, $k_{0}$ and $\sigma_{0}$ are respectively the maximum stiffness and contraction. $n_{0}$ is a reduction factor that allows to take into account the Starling effect by which the maximum contraction depends on the fibre strain $e_{c}$. The control variable $u$ is derived from the electrical activation model and is a function of the free calcium concentration only. It is modeled using electrophysiological inputs such as depolarization times $\left(T_{d}\right)$ and action potential durations $(A P D)$ and depends on two parameters: $k_{A T P}$ the rate of the myosin ATPase activity controlling the contraction rate and $k_{R S}$ the rate of sarcoplasmic reticulum calcium re-uptake controlling the relaxation rate.

The ventricles are filled with blood coming from the atria and ejected through the arteries. A valve model gives relationships between the outward flow and the various pressures (ventricular, arteria and atria). The arteria pressure is modeled using a four-element Windkessel model (Stergiopulos et al, 1999), that depends on four parameters: the peripheral resistance $R_{p}$, the characteristic time $\tau$, the characteristic resistance $Z_{c}$ and the total arteria inertance $L$.

The values of the mentioned parameters are given Table.1.

Table 1: Parameter values.

\begin{tabular}{llr}
\hline Notation & Parameter Name & Value \\
\hline$\sigma_{0}(\mathrm{MPa})$ & Max Contraction & 7 \\
$k_{0}(\mathrm{MPa})$ & Max Stiffness & 5 \\
$k_{A T P}\left(\mathrm{~s}^{-1}\right)$ & Contraction Rate & 15 \\
$k_{R S}\left(\mathrm{~s}^{-1}\right)$ & Relaxation Rate & 35 \\
$E_{s}(\mathrm{MPa})$ & Linear Modulus & 10 \\
$\alpha$ & Cross-bridges Unfasten Rate & 0.5 \\
$\mu(\mathrm{MPa} . \mathrm{s})$ & Viscosity & 1.3 \\
$c_{1}(\mathrm{kPa})$ & Mooney Rivlin Modulus & 10 \\
$c_{2}(\mathrm{kPa})$ & Mooney Rivlin Modulus & 10 \\
$K(\mathrm{MPa})$ & Bulk Modulus & 10 \\
$\tau(s)$ & Wind. Charact. Time & 0.8 \\
$R_{p}\left(\mathrm{MPa} \cdot \mathrm{m}^{-3} \cdot \mathrm{s}\right)$ & Wind. Periph. Resistance & 100 \\
$Z_{c}\left(\mathrm{MPa} \cdot \mathrm{m}^{-3} \cdot \mathrm{s}\right)$ & Wind. Charact. Resistance & 1 \\
$L\left(\mathrm{kPa} \cdot \mathrm{s}^{2} \cdot \mathrm{m}^{-3}\right)$ & Wind. Total Art. Inertance & 10 \\
\hline
\end{tabular}

\section{References}

Aliev R, Panfilov A (1996) A simple two-variable model of cardiac excitation. Chaos, Solitons \& Fractals 7(3):293-301

Attene M, Falcidieno B (2006) ReMESH: An interactive environment to edit and repair triangle meshes. In: Shape Modeling and Applications, IEEE International Conference on, $\mathrm{p} 41$

Baxley W, Joseph Reeves T (1971) Abnormal regional myocardial performance in coronary artery disease. Progress in cardiovascular diseases 13(5):405-421

Bayer J, Blake R, Plank G, Trayanova N (2012) A novel rulebased algorithm for assigning myocardial fiber orientation to 
computational heart models. Annals of Biomedical Engineering pp 1-12

Bestel J, Clement F, Sorine M (2001) A biomechanical model of muscle contraction. Medical Image Computing and Computer Assisted Intervention (MICCAI) pp 1159-1161

Billet F (2010) Assimilation de données images pour la personnalisation d'un modèle électromécanique du coeur. PhD thesis, Université de Nice -Sophia Antipolis, in French

Butakoff C, Sukno F, Doltra A, Silva E, Sitges M, Frangi A (2011) Order statistic based cardiac boundary detection in $3 d+t$ echocardiograms. FIMH pp 359-366

Cazeau S, Leclercq C, Lavergne T, Walker S, Varma C, Linde C, Garrigue S, Kappenberger L, Haywood G, Santini M, et al (2001) Effects of multisite biventricular pacing in patients with heart failure and intraventricular conduction delay. New England Journal of Medicine 344(12):873-880

Chabiniok R, Moireau P, Lesault P, Rahmouni A, Deux J, Chapelle D (2011) Estimation of tissue contractility from cardiac cine-MRI using a biomechanical heart model. Biomechanics and Modeling in Mechanobiology pp 1-22

Chapelle D, Fragu M, Mallet V, Moireau P (2012a) Fundamental principles of data assimilation underlying the verdandi librairy: applications to biophysical model personalization with euheart. Medical \& Biological Engineering \& Computing (MBEC)

Chapelle D, Le Tallec P, Moireau P, Sorine M (2012b) An energy-preserving muscle tissue model: formulation and compatible discretizations. International Journal for Multiscale Computational Engineering 10(2):189-211

Chung E, Leon A, Tavazzi L, Sun J, Nihoyannopoulos P, Merlino J, Abraham W, Ghio S, Leclercq C, Bax J, et al (2008) Results of the predictors of response to crt (prospect) trial. Circulation 117(20):2608-2616

Clayton RH, Bernus O, Cherry EM, Dierckx H, Fenton FH, Mirabella L, Panfilov AV, Sachse FB, Seemann G, Zhang H (2011) Models of cardiac tissue electrophysiology: progress, challenges and open questions. Prog Biophys Mol Biol 104(13):22-48

De Craene M, Piella G, Camara O, Duchateau N, Silva E, Doltra A, D'hooge J, Brugada J, Sitges M, Frangi AF (2012a) Temporal diffeomorphic free-form deformation: Application to motion and strain estimation from 3D echocardiography. Med Image Anal 16(2):427-450

De Craene M, Tobon-Gomez C, Butakoff C, Duchateau N, Piella G, Rhode KS, Frangi AF (2012b) Temporal diffeomorphic free form deformation (TDFFD) applied to motion and deformation quantification of tagged MRI sequences. In: STACOM, Lecture Notes in Computer Science, vol 7085, pp 6877

Delingette H, Billet F, Wong K, Sermesant M, Rhode K, Ginks M, Rinaldi C, Razavi R, Ayache N (2012) Personalization of cardiac motion and contractility from images using variational data assimilation. Biomedical Engineering, IEEE Transactions on 59(1):20-24

Ecabert O, Peters J, Walker MJ, Ivanc T, Lorenz C, von Berg J, jonathan Lessick, mani Vembar, Weese J (2011) Segmentation of the heart and great vessels in CT images using a model-based adaptation framework. Medical Image Analysis 15(6):863-876

FitzHugh R (1961) Impulses and physiological states in theoretical models of nerve membrane. Biophysical Journal 1:445466

Hodgkin A, Huxley A (1952) A quantitative description of membrane current and its application to conduction and excitation in nerve. Journal of Physiology 177:500-544

Hsu EW, Henriquez CS (2001) Myocardial fiber orientation mapping using reduced encoding diffusion tensor imaging.
Journal of Cardiovascular Magnetic Resonance 3(4):339-347

Humphrey J, Strumpf R, Yin F (1990) Determination of a constitutive relation for passive myocardium: I. a new functional form. Journal of biomechanical engineering 112:333

Hunter P, Nash M, Sands G (1997) Computational electromechanics of the heart. Computational biology of the heart pp 345-407

Huxley A (1957) Muscle structure and theories of contraction. Progress in biophysics and biophysical chemistry 7:255

Imperiale A, Chabiniok R, Moireau P, Chapelle D (2011) Constitutive parameter estimation using tagged-mri data. In: Proceedings of FIMH'11, LNCS 6666, Springer, pp 409-417

Julier S, Uhlmann J (1997) A new extension of the Kalman filter to nonlinear systems. In: Int. Symp. Aerospace/Defense Sensing, Simul. and Controls, Citeseer, vol 3, p 26

Koon K, Thebault C, Le Rolle V, Donal E, Hernndez A (2010) Atrioventricular delay optimization in cardiac resynchronization therapy assessed by a computer model. In: Computing in Cardiology, 2010, pp $333-336$

Lombaert H, Peyrat JM, Croisille P, Rapacchi S, Fanton L, Clarysse P, Delingette H, Ayache N (2011) Statistical Analysis of the Human Cardiac Fiber Architecture from DT-MRI. In: Axel L, Metaxas D (eds) Proceedings of FIMH Conference 2011, Springer, LNCS, vol 6666, pp 171-179, best Paper Award

Lorensen WE, Cline HE (1987) Marching cubes: A high resolution $3 \mathrm{~d}$ surface construction algorithm. Computer Graphics 21(4):163-169

Mansi T, Pennec X, Sermesant M, Delingette H, Ayache N (2011) iLogDemons: A demons-based registration algorithm for tracking incompressible elastic biological tissues. International Journal of Computer Vision 92(1):92-111

Marchesseau S, Delingette H, Sermesant M, Ayache N (2012a) Fast parameter calibration of a cardiac electromechanical model from medical images based on the unscented transform. Biomechanics and Modeling in Mechanobiology pp 117

Marchesseau S, Delingette H, Sermesant M, Rhode K, Duckett S, Rinaldi C, Razavi R, Ayache N (2012b) Cardiac mechanical parameter calibration based on the unscented transform. In: Medical Image Computing and Computer Assisted Intervention (MICCAI), Springer, LNCS, vol 7511, best paper award

Marchesseau S, Delingette H, Sermesant M, Sorine M, Rhode K, Duckett S, Rinaldi C, Razavi R, Ayache N (2012c) Preliminary Specificity Study of the Bestel-Clément-Sorine Electromechanical Model of the Heart using Parameter Calibration from Medical Images. Journal of the Mechanical Behavior of Biomedical Materials . In Press

McLeod K, Prakosa A, Mansi T, Sermesant M, Pennec X (2012) An Incompressible Log-Domain Demons Algorithm for Tracking Heart Tissue. In: Proc. MICCAI Workshop on Statistical Atlases and Computational Models of the Heart: Mapping Structure and Function (STACOM11), Springer, Toronto, LNCS, vol 7085, pp 54-65

Mitchell CC, Schaeffer DG (2003) A two-current model for the dynamics of cardiac membrane. Bulletin of mathematical biology 65(5):767-793

Moireau P, Chapelle D (2011) Reduced-order Unscented Kalman Filtering with application to parameter identification in large-dimensional systems. ESAIM: Control Optimisation and Calculus of Variations 17:380-405, doi:10.1051/cocv/2010006

Moireau P, Chapelle D, Tallec PL (2008) Joint state and parameter estimation for distributed mechanical systems. Computer Methods in Applied Mechanics and Engineering 197:659_ 677 
Nash M (1998) Mechanics and material properties of the heart using an anatomically accurate mathematical model. $\mathrm{PhD}$ thesis, University of Auckland

Noble D (1962) A modification of the hodgkin-huxley equations applicable to purkinje fibre action and pace-maker potentials. J Physiol 160:317-352

Pashaei A, Romero D, Sebastian R, Camara O, Frangi A (2011) Fast multiscale modelling of cardiac electrophysiology including purkinje system. Biomedical Engineering, IEEE Transactions on (99):1-1

Petitjean C, Dacher JN (2011) A review of segmentation methods in short axis cardiac mr images. Medical Image Analysis pp 169-184

Relan J, Chinchapatnam P, Sermesant M, Rhode K, Ginks M, Delingette H, Rinaldi CA, Razavi R, Ayache N (2011) Coupled Personalization of Cardiac Electrophysiology Models for Prediction of Ischaemic Ventricular Tachycardia. Journal of the Royal Society Interface Focus 1(3):396-407

Sachse F (2004) Computational cardiology: modeling of anatomy, electrophysiology, and mechanics, vol 2966. Springer Verlag

Sainte-Marie J, Chapelle D, Cimrman R, Sorine M (2006) Modeling and estimation of the cardiac electromechanical activity. Computers \& structures 84(28):1743-1759

Schaerer J, Casta C, Pousin J, Clarysse P (2010) A dynamic elastic model for segmentation and tracking of the heart in $\mathrm{mr}$ image sequences. Medical Image Analysis pp 738-749

Sermesant M, Konukoglu E, Delingette H, Coudiere Y, Chinchaptanam P, Rhode K, Razavi R, Ayache N (2007) An anisotropic multi-front fast marching method for real-time simulation of cardiac electrophysiology. In: Proceedings of Functional Imaging and Modeling of the Heart 2007 (FIMH'07), LNCS, vol 4466, pp 160-169

Sermesant M, Chabiniok R, Chinchapatnam P, Mansi T, Billet F, Moireau P, Peyrat J, Wong K, Relan J, Rhode K, Ginks M, Lambiase P, Delingette H, Sorine M, Rinaldi C, Chapelle D, Razavi R, Ayache N (2012) Patient-specific electromechanical models of the heart for the prediction of pacing acute effects in CRT: A preliminary clinical validation. Medical Image Analysis 16(1):201-215

Smith N, de Vecchi A, McCormick M, Nordsletten D, Camara O, Frangi A, Delingette H, Sermesant M, Relan J, Ayache N, Krueger MW, Schulze W, Hose R, Valverde I, Beerbaum P, Staicu C, Siebes M, Spaan J, Hunter P, Weese J, Lehmann H, Chapelle D, Razavi R (2011) euheart: Personalized and integrated cardiac care using patient-specific cardiovascular modelling. Journal of the Royal Society Interface Focus 1(3):349364

Stergiopulos N, Westerhof B, Westerhof N (1999) Total arterial inertance as the fourth element of the windkessel model. American Journal of Physiology-Heart and Circulatory Physiology 276(1):H81

Streeter DD (1979) Gross morphology and fiber geometry of the heart. Handbook of physiology pp 61-112

Tobon-Gomez C, Sukno FM, Butakoff C, Huguet M, Frangi AF (2012) Automatic training and reliability estimation for 3D ASM applied to cardiac MRI segmentation. Phys Med Biol 57(13): 4155

Tobon-Gomez C, De Craene M, McLeod K, Tautz L, Shi W, Hennemuth A, Prakosa A, Wang H, Carr-White G, Kapetanakis S, Lutz A, Rasche V, Schaeffter T, Butakoff C, Friman O, Mansi T, Sermesant M, Zhuang X, Ourselin S, Peitgen HO, Pennec X, Razavi R, Rueckert D, Frangi AF, Rhode KS (In Press) Benchmarking framework for myocardial tracking and deformation algorithms: An open access database. Medical Image Analysis
Wang V, Lam H, Ennis D, Cowan B, Young A, Nash M (2009) Modelling passive diastolic mechanics with quantitative MRI of cardiac structure and function. Medical Image Analysis 13(5):773-784

Xi J, Lamata P, Lee J, Moireau P, Chapelle D, Smith N (2011) Myocardial transversely isotropic material parameter estimation from in-silico measurements based on a reduced-order unscented kalman filter. Journal of the mechanical behavior of biomedical materials 4(7):1090-1102

Zheng Y, Barbu A, Georgescu B, Scheuering M, Comaniciu D (2008) Four-chamber heart modeling and automatic segmentation for 3-d cardiac ct volumes using marginal space learning and steerable features. IEEE Trans Med Imaging pp 1668-1681

Zhu Y, Papademetris X, Sinusas A, Duncan JS (2010) A coupled deformable model for tracking myocardial borders from realtime echocardiography using an incompressibility constraint. Medical Image Analysis pp 429-448 